\title{
Systematic review of gastrostomy complications and outcomes in pediatric cancer and bone marrow transplant
}

\author{
James Evans MRes ${ }^{1,2}$ (1) | Breeana Gardiner BSc ${ }^{1} \quad$ Dan Green PhD \\ Faith Gibson $\mathbf{P h D}^{4,5} \quad$ Graeme O’Connor $\mathbf{P h D}^{1,2} \quad$ Julie Lanigan $\mathbf{P h D}^{2}$ \\ ${ }^{1}$ Dietetics Department, Great Ormond Street Hospital for Children, London, UK \\ ${ }^{2}$ University College London Great Ormond Street Institute of Child Health, London, UK \\ ${ }^{3}$ Section of Public Health, School of Health and Related Research (ScHARR), University of Sheffield, Sheffield, UK \\ ${ }^{4}$ School of Health Sciences, University of Surrey, Guildford, Surrey, UK \\ ${ }^{5}$ Centre for Outcomes and Experience Research in Children's Health, Illness and Disability (ORCHID), Great Ormond Street Hospital for Children, \\ London, UK
}

\section{Correspondence}

James Evans, MRes, Dietetics Department, Great Ormond Street Hospital for Children, Great Ormond Street, London WC1N 3JH, UK.

Email:james.evans@gosh.nhs.uk

\begin{abstract}
Background: Nutrition support is essential in children with cancer, including those undergoing bone marrow transplant (BMT), to reduce the risk of malnutrition and associated deleterious outcomes. Enteral nutrition is more commonly provided via nasogastric than gastrostomy tubes because of safety concerns with the latter in immunocompromised children. This systematic review investigated the incidence and type of complications and outcomes in pediatric cancer patients fed by gastrostomy.

Methods: Databases were searched for randomized and observational studies investigating the use of any gastrostomy device in children aged $<18$ years with any cancer diagnosis, including those undergoing BMT. Five cohort and 11 case series studies were included. Owing to clinical heterogeneity, meta-analyses were not performed.

Results: Quality of evidence varied, with five studies judged at serious risk of bias and poor quality; however, the remaining 11 were considered to range from moderate to good quality. Across studies, $54.6 \%$ of children developed one or more complications, of which $76.6 \%$ were classified as minor, $23.4 \%$ major. The most frequent complications included inflammation (52\% of episodes), infection (42.1\%), leakage (22.3\%), and granuloma (21\%). Evidence regarding infection rates in cancer/BMT patients compared with other disease states was inconclusive. Gastrostomy feeding was associated with improvement or stabilization of nutrition status in $77 \%-92.7 \%$ of children.
\end{abstract}

This is an open access article under the terms of the Creative Commons Attribution License, which permits use, distribution and reproduction in any medium, provided the original work is properly cited.

(c) 2021 The Authors. Nutrition in Clinical Practice published by Wiley Periodicals LLC on behalf of American Society for Parenteral and Enteral Nutrition 
Conclusion: Gastrostomy feeding in this population is relatively safe and effective in stabilizing or improving nutrition status throughout treatment. Complications are frequent but mostly minor. Placement requires careful consideration of the complications, benefits, nutrition risk and status at diagnosis, and quality of life.

\section{KEYWOR D S}

bone marrow transplantation, cancer, enteral nutrition, gastrostomy, pediatrics, systematic review

\section{INTRODUCTION}

Malnutrition is a risk for children with cancer, including those undergoing bone marrow transplant (BMT), with the prevalence estimated at 5\%-50\%. ${ }^{1}$ Malnutrition has been associated with reduced therapy tolerance, delayed wound healing, increased risk of infection, longer hospital stay, higher relapse rates, worse quality of life, and graft-vs-host disease (GvHD), compared with those who are adequately nourished at diagnosis and maintain a good nutrition status throughout treatment, in pediatric cancer and BMT. ${ }^{2-8}$ The risk of malnutrition is higher in children with advanced disease, metastatic solid tumors, and higher treatment intensity, such as in highrisk neuroblastoma, medulloblastoma, and BMT, compared with nonmetastatic solid tumors and acute lymphoblastic leukemia treated with regimen A. ${ }^{9}$ Treatment side effects include nausea, vomiting, anorexia, diarrhea, and mucositis, which negatively impact nutrition intake. ${ }^{10}$ Combining these with metabolic and psychological factors exacerbates the risk of developing malnutrition. ${ }^{11}$ Childhood is a critical period for growth, bone formation, and brain development. ${ }^{12}$ Consequently, optimal nutrition is essential to support growth and development and meet the additional needs of disease. ${ }^{9}$

There are no international guidelines for nutrition support in pediatric cancer and BMT. However, the American Society for Parenteral and Enteral Nutrition (ASPEN) and the European Society for Clinical Nutrition and Metabolism (ESPEN) published guidelines for the nutrition support of adult cancer and BMT patients in 2009 and 2021, respectively. ${ }^{13,14}$ These recommend enteral nutrition as the first option for nutrition support in patients with a functioning gastrointestinal tract. Enteral tube feeding is an effective and safe method to minimize weight loss during treatment, ${ }^{15}$ improve nutrition status and energy intake during intensive treatment with minimal complications, ${ }^{16,17}$ and maintain mucosal gut integrity, thereby reducing bacterial translocation. ${ }^{18}$
In this population, enteral nutrition is most commonly provided via nasogastric tubes (NGTs). ${ }^{18,19}$ However, NGTs are susceptible to complications, including discomfort (particularly with mucositis), dislodgment with vomiting, placement refusal, and contraindication due to mechanical obstruction in the nasopharynx. ${ }^{18,20,21}$ These complications can hinder the provision of adequate enteral nutrition. Gastrostomy tubes avoid some of these complications. They are fixed in place and so are less susceptible to dislodgment; can be hidden under clothes, making them maybe more aesthetically acceptable ${ }^{21}$; and have been associated with less use of parenteral nutrition compared with use by children fed via NGT during BMT. ${ }^{22}$ However, gastrostomy tubes have not commonly been used in immunocompromised children because of the risk of infectious complications. ${ }^{23}$ Although complications associated with gastrostomy feeding, including infection, have been shown in BMT $^{24}$ and inflammatory skin events, skin erosion, and granuloma have been shown in cancer populations, ${ }^{17,25}$ other studies in this area have shown weight gain and only minor complications. $^{26,27}$

In this article, we systematically reviewed the evidence relating to the complications and outcomes of gastrostomy use in this population. Findings will help guide clinicians' decision-making when considering options to provide enteral nutrition. A narrative review investigating indications, contraindications, complications, and management of gastrostomy tubes in children with cancer was published in 2019. ${ }^{21}$ However, a scoping search of the international prospective register of systematic reviews (PROSPERO) in July 2020 identified no systematic reviews previously published or underway in this area.

The primary objective of this review was to investigate the incidence and type of complications associated with gastrostomy use in children with cancer, including those undergoing BMT. Secondary aims were to evaluate the efficacy of gastrostomies in relation to various nutrition and clinical outcomes. 


\section{METHODS}

\section{Protocol registration}

The protocol for this review was written according to the Preferred Reporting Items for Systematic Review and Meta-Analyses Protocols (PRISMA-P) ${ }^{28}$ and registered with PROSPERO on September 28, 2020: CRD42020211700. This systematic review adheres to the PRISMA guidelines. ${ }^{29}$

\section{Eligibility criteria}

We included randomized controlled trials, controlled (nonrandomized) clinical trials, prospective and retrospective cohort studies, case-control, and case series. Gray literature was included, if appropriate. Qualitative and animal studies, reviews, commentaries, and studies without full-text availability were excluded. Authors of abstracts for which a full-text article could not be found were contacted to see if a full-text report existed. No restrictions were imposed regarding date, country, language, or setting of studies. Non-English studies were translated by known healthcare professionals within our hospital.

Participants included children ( $<18$ years, although we accepted studies with an age range up to 21 years when the average age was below 18) with any cancer diagnosis receiving any treatment, including those undergoing BMT from any donor or cell source for any diagnosis. If a study included a mixed population-for example, of adults and children-it was included if children's data were reported separately. If data were not reported in this way, authors were contacted to request this information. The intervention was the use of any gastrostomy device placed using any technique.

Comparators were not essential for this review. Studies with no comparator were included, as these would provide evidence on complications and outcomes of gastrostomy use. Comparators could include children with cancer/BMT receiving nutrition support through various routes, including NGT or parenteral nutrition. Regarding complications, a comparator could include gastrostomy use in noncancer/non-BMT disease states. Children with neurological impairment have been considered the "reference population" in this circumstance ${ }^{17}$ because they will not likely become immunocompromised, as is common in those receiving cancer treatment/BMT, which poses a risk for infectious complications.

Primary outcomes included the incidence and type of any gastrostomy complications, investigated at any time point until gastrostomy removal. Secondary outcomes included a wide range of nutrition and clinical parameters, including nutrition intakes, anthropometry, survival, and length of admission, that result from gastrostomy use, as well as any comparator methods of nutrition support that studies may have used.

\section{Information sources and search}

Databases searched via Ovid included MEDLINE (1946 onward), Embase (1974 onward), Cochrane Central Register of Controlled Trials, AMED (1985 onward), and CINAHL (via EBSCO). Gray literature searched included EThOS (ethos.bl.uk) and Open Grey (www.opengrey.eu). To strive for literature saturation, clinical experts were contacted in September 2020 for any further articles that could have been missed from database searches, and reference lists of included studies, relevant reviews, ${ }^{30,31}$ and the table of contents of key journals Pediatric Blood \& Cancer and Journal of Pediatric Hematology/Oncology from January 2019 (dates since the most recent nonsystematic review) ${ }^{21}$ were scanned.

The search strategy was developed in consultation with a Health Sciences Research Librarian. One author (J.E.) searched all sources. The final MEDLINE (Ovid) search strategy (Supplementary Material S1) was adapted for use with other databases. The last search was run on September 18, 2020.

\section{Study selection}

Studies were managed using Covidence ${ }^{32}$ and references using Mendeley. ${ }^{33}$ Search results were imported from databases into Covidence and duplicates removed and then checked manually to remove missed duplicates and multiple study reporting. References were independently screened by two authors (J.E. and B.G.) against the eligibility criteria, first on title and abstract and second on fulltext reports of studies that appeared eligible. When necessary, study authors were contacted to resolve uncertainty around eligibility.

\section{Data items and extraction}

Data were extracted independently by two authors (J.E. and B.G.) using a custom extraction Excel spreadsheet. Data were extracted on general information (author, country, funding, conflicts of interest, study design), population (inclusion/exclusion criteria, patient characteristics, sample size), interventions (type of gastrostomy), comparators, 
(other nutrition support interventions), and outcomes (gastrostomy complications and classification, time points, nutrition and clinical parameters). Study authors were contacted up to a maximum of three times via email to resolve any uncertainties or obtain missing information.

\section{Risk-of-bias assessment}

The search identified only observational studies. Therefore, the Risk of Bias in Non-randomized Studies of Interventions (ROBINS-I) ${ }^{34}$ tool was used to assess studies in which participants were not randomized to comparison groups. Domains include confounding, participant selection, classification of interventions, deviations from intended interventions, missing data, measurement of outcomes, and selection of reported results. Bias in each domain was classified as "low," "moderate," "serious," "critical," or "no information" according to responses to signaling questions. Each study was given an overall risk of bias equivalent to the most severe level in any domain. Studies without comparison groups were assessed using the National Institutes of Health (NIH) quality assessment tool for before-after (pre-post) studies with no control group. ${ }^{35}$ Criteria include how clearly the research question, population, intervention, outcomes, statistical methods, sample size, and results are described. Each study was given an overall rating of "good," "fair," or "poor." The risk of bias was assessed independently by two authors (J.E. and B.G.) at the study level. Disagreements regarding study selection, data extraction, and risk-of-bias assessment were resolved by consensus between the two authors, with a third author (G.O.C.) acting as arbiter, if required.

Included studies that compared groups were clinically heterogeneous regarding populations, interventions, and outcomes. Therefore, meta-analyses were not undertaken, and results are presented in detailed tables and narrative form.

\section{RESULTS}

\section{Included studies}

Database searches yielded 8240 studies. Two additional studies were identified through other sources. After removing duplicates, 7664 studies remained. Following screening on title and abstract, 7592 were excluded for not meeting the eligibility criteria. The full text of the remaining 72 studies was screened for eligibility. Of these, 16 articles with data on 681 children with gastrostomies were included (Figure 1).

\section{Study characteristics}

One study was translated from French ${ }^{36}$; others were in English. All but two studies ${ }^{17,36}$ were single-center, with $50 \%$ ( 8 of 16) undertaken in Europe, ${ }^{17,22,26,36-40} 44 \%$ (7 of 16) in North America, ${ }^{24,25,27,41-44}$ and one in Australia. ${ }^{45}$ Study inclusion periods ranged from 3.5 to 16 years, ${ }^{25,26}$ with average ages from 2 to 13 years. ${ }^{24,36,40}$ Two studies focused on children undergoing BMT, ${ }^{22,24}$ three investigated mixed populations undergoing cancer treatment with and without BMT, ${ }^{26,27,38}$ and 69\% (11 of 16) included children undergoing treatment for various malignant diseases without BMT.

No studies were randomized, 69\% (11 of 16) were case series following one group only (seven were retrospective, ${ }^{25,27,38,40,43-45}$ whereas four did not state whether they were retrospective or prospective $\left.\mathrm{e}^{26,37,41,42}\right)$, and $31 \%$ (5 of 16) were cohort studies ${ }^{17,22,24,36,39}$ comparing two groups (only one was prospective ${ }^{39}$ ). Comparator groups included children with gastrostomies for noncancer/non-BMT reasons (typically neurological impairment ${ }^{17,24,39}$ ) or NGT during cancer treatment without $^{17}$ or with BMT. ${ }^{22}$ Interventions varied across studies; $50 \%$ (8 of 16) used percutaneous endoscopic gastrostomies $^{17,22,24,26,36,38,43,45}$; 31\% (5 of 16) used mixed devices and insertion techniques, including buttons, surgical, open and interventional radiology, $25,27,41,42,44$ with others using percutaneous radiologic-, ${ }^{40}$ video-,${ }^{39}$ or laparoscopy-aided buttons. ${ }^{37}$ Four studies compared complications by insertion technique and device. ${ }^{25,41,42,44}$

Gastrostomies were placed prophylactically to preempt nutrition problems during $\mathrm{BMT}^{22,24}$ or mucositis ${ }^{45}$ or in children with preexisting malnutrition who are therefore at high nutrition risk at diagnosis. ${ }^{17,36,39,40}$ Other indications included anorexia, reduced dietary intake and weight loss during treatment, and unsafe oral feeding. ${ }^{26,27,38,41}$ Studies reported gastrostomy insertion ranging from 1.5 to $7.4^{17,26,27,45}$ months after diagnosis. In two studies, they were placed $22^{22}$ and $56.5^{24}$ days pre-BMT. The average duration of gastrostomy use was 10 months (range, 6.3-12.9 $9^{25,26}$ ). All studies reported gastrostomy complications, 11 reported various clinical and nutrition outcomes. Included studies are summarized in Table S1.

\section{Study quality}

Studies comparing groups were assessed as being at serious ${ }^{17,36}$ and moderate ${ }^{22,24,39}$ risk of bias using ROBINS-I. ${ }^{34}$ Studies with no comparator were assessed as poor, ${ }^{26,37,41}$ fair, ${ }^{38,42,45}$ or good ${ }^{25,27,40,43,44}$ quality using the $\mathrm{NIH}$ tool. ${ }^{35}$ Common methodological weaknesses 


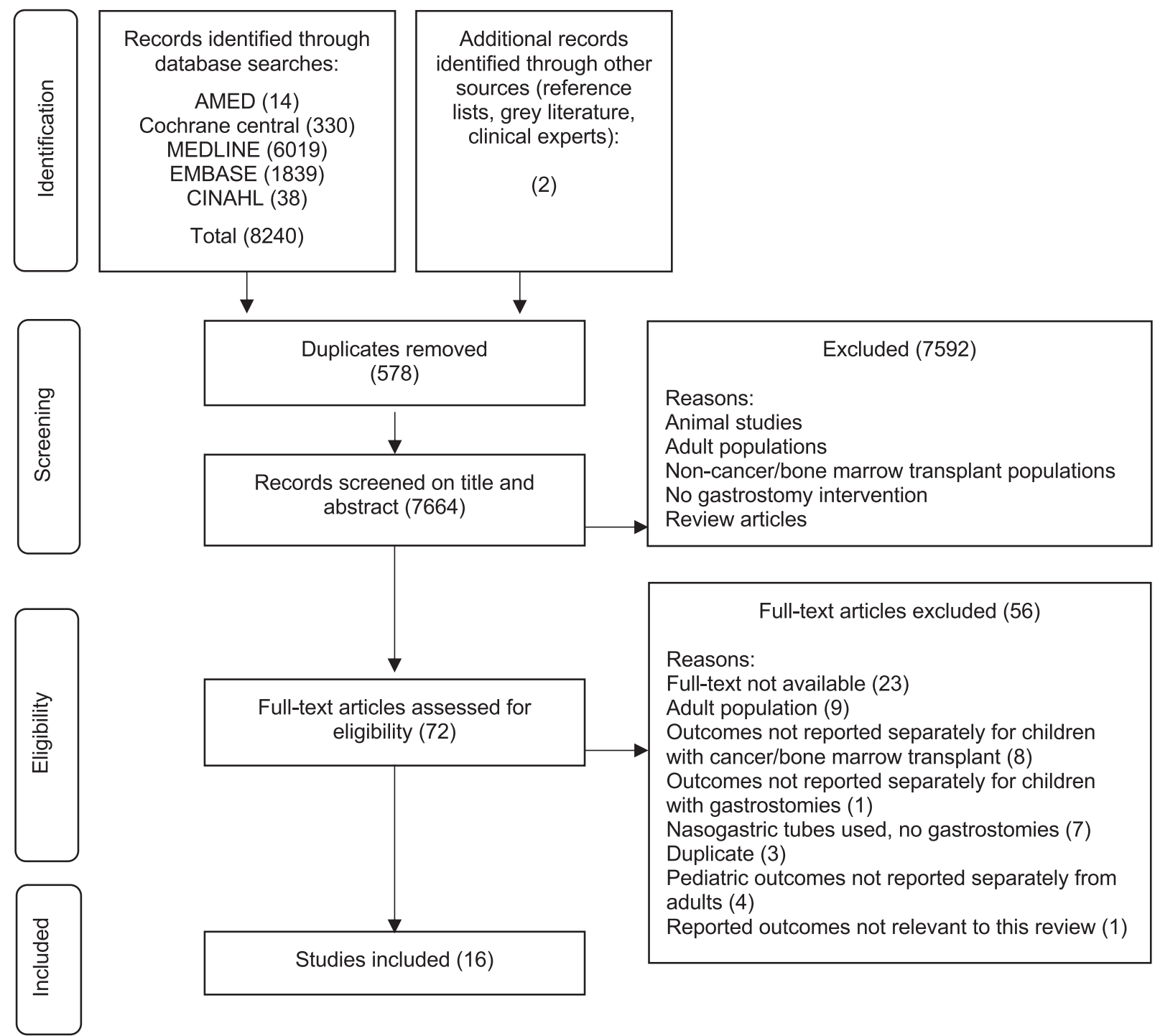

F I G U R E 1 Preferred Reporting Items for Systematic Review and Meta-Analyses (PRISMA) flow diagram showing studies included in the systematic review

included the absence of randomization, retrospective study designs limiting the accuracy and reliability of data (particularly complications), small samples (no studies provided sample size/power calculations), lack of detail regarding participant selection, and outcomes that were not clearly prespecified. The risk-of-bias assessments are shown in Tables S2 and S3.

\section{Incidence and timing of complications}

Complications were categorized as major or minor in 69\% (11 of 16) of studies according to each author's own criteria (Table S4). Across all studies, 54.6\% $(n=351)$ of patients developed one or more complications, of which $27.4 \%$ $(n=46)$ developed a major complication. Of complication episodes, $23.4 \%(n=88)$ were major, and $76.6 \%(n=472)$ were minor (Table 1). In the 11 studies on children with cancer not undergoing BMT, $59.4 \%(n=336)$ of patients developed one or more complications, of which $12.2 \%(n=$ 32) developed a major complication. Of the episodes, $7.3 \%$ $(n=74)$ were major, and $92.7 \%(n=471)$ were minor. Of the two BMT studies, ${ }^{22,24} 28.4 \%(n=15)$ of patients developed one or more complications, of which $95.5 \%(n=14)$ developed a major complication. Of the episodes, $95.5 \%$ $(n=14)$ were major. No minor complications occurred in one study, ${ }^{24}$ whereas $9.1 \%(n=1)$ were minor in the other.

One study reported complications according to underlying cancer diagnosis, with $63 \%(n=64)$ of patients having central nervous system tumors, $60 \%(n=27)$ with solid tumors, and $60 \%(n=15)$ with leukemia/lymphoma developing a complication. ${ }^{25}$

Studies reported complications at differing time points after gastrostomy placement. Complications were seen 

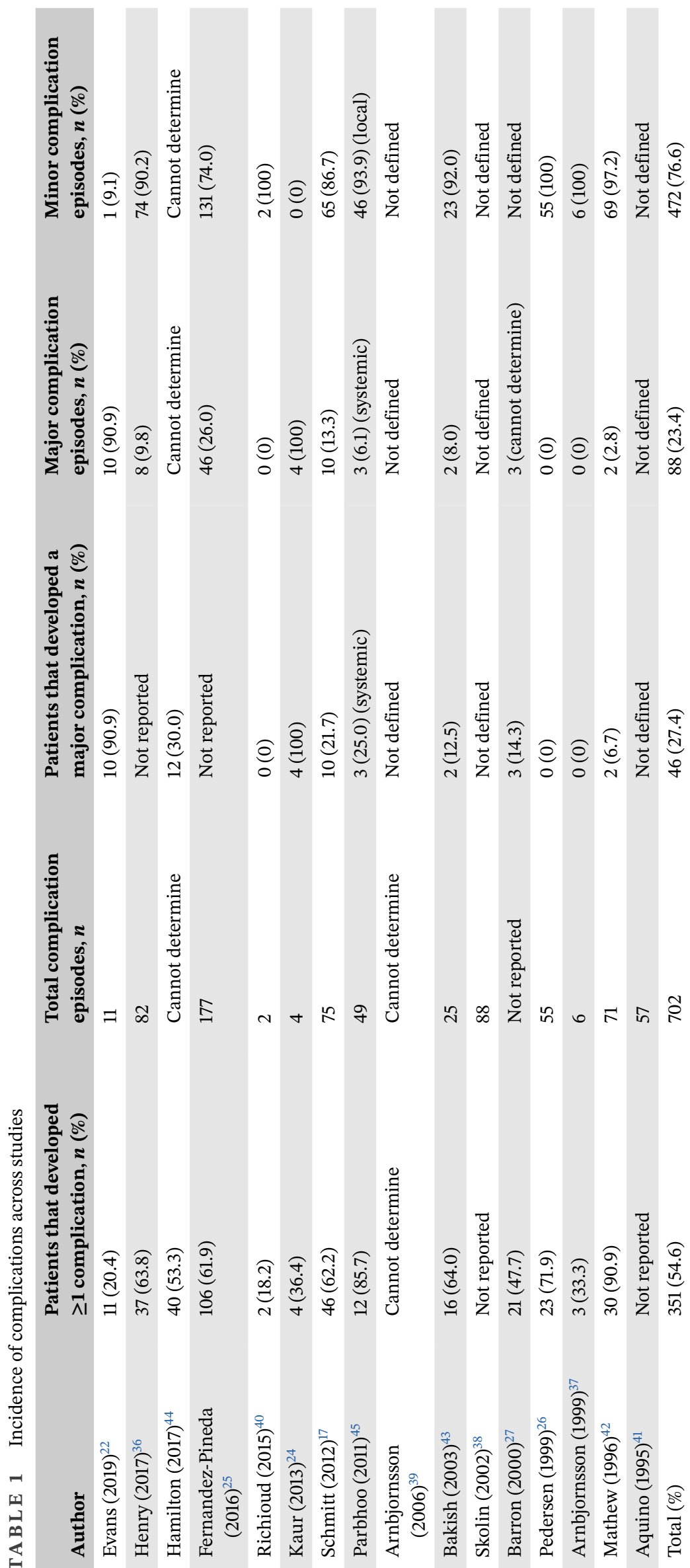
TA B LE 2 Incidence of gastrostomy site infectious complications

\begin{tabular}{|llll|}
\hline Author & $\begin{array}{l}\text { Peritubal wound } \\
\text { infection, } \boldsymbol{n} \text { episodes } \\
\text { total episodes) }\end{array}$ & $\begin{array}{l}\text { Peritubal wound } \\
\text { infection, } \boldsymbol{n} \text { patients }(\% \\
\text { total patients) }\end{array}$ & $\begin{array}{l}\text { Peritubal wound } \\
\text { inflammation, } \boldsymbol{n} \text { episodes (\% } \\
\text { total episodes) }\end{array}$ \\
\hline Evans $(2019)^{22}$ & $11(100)$ & $11(20.4)$ & Not reported \\
\hline Henry $(2017)^{36}$ & $16(19.5)$ & Not reported & $63(76.8)$ \\
\hline Hamilton $(2017)^{44}$ & Not reported & Not reported & Not reported \\
\hline Fernandez-Pineda $(2016)^{25}$ & $42(23.7)$ & Not reported & Not reported \\
\hline Richioud $(2015)^{40}$ & $0(0)$ & $0(0)$ & Not reported \\
\hline Kaur $(2013)^{24}$ & $4(100)$ & $4(36.4)$ & Not reported \\
\hline Schmitt $(2012)^{17}$ & $6(8.0)$ & Not reported & $29(38.7)$ \\
\hline Parbhoo $(2011)^{45}$ & $16(32.7)$ & $9(64.3)$ & $4(8.2)$ \\
\hline Arnbjornsson $(2006)^{39}$ & $5($ cannot determine) & Not reported & Not reported \\
\hline Bakish $(2003)^{43}$ & $23(92.0)$ & $15(60.0)$ & Not reported \\
\hline Skolin $(2002)^{38}$ & $57(64.8)$ & $18(100)$ & Not reported \\
\hline Barron $(2000)^{27}$ & Not reported & $18(40.9)$ & Not reported \\
\hline Pedersen $(1999)^{26}$ & $23(41.8)$ & $12(37.5)$ & Not reported \\
\hline Arnbjornsson $(1999)^{37}$ & $0(0)$ & $0(0)$ & Not reported \\
\hline Mathew $(1996)^{42}$ & Not reported & $15(45.5)$ & 23 patients $(69.7)$ \\
\hline Aquino $(1995)^{41}$ & $13(22.8)$ & $9(36.0)$ & $38(66.7)$ \\
\hline Total $(\%)$ & $216(42.1)$ & $111(40.1)$ & $157(52.0)$ \\
\hline
\end{tabular}

within 1 week of placement in $21.4 \%^{45}$ and $50 \%^{24}$ of patients, within 1 month in $18.5 \%$ of patients ${ }^{39}$ and $20.3 \%$ of episodes, ${ }^{25}$ within 2 months in $100 \%$ of patients, ${ }^{24}$ and within 6 months in $29.6 \%$ of patients. ${ }^{39}$

\section{Mechanical complications}

Mechanical and other noninfectious gastrostomy complications accounted for a small proportion of total episodes, including buried bumper syndrome, bleeding, gastrocutaneous fistula, tube occlusion, dislodgment, migration, breakage (all <7\%), pain/discomfort $(15.8 \%)$, granuloma (21.0\%), and peristomal leakage (22.3\%) (Table S5).

\section{Infectious complications}

Infection and inflammation made up the largest proportion of total episodes ( $42.1 \%$ and $52.0 \%$, respectively), and $40.1 \%$ of patients with a gastrostomy experienced an infection (Table 2). Across the 11 studies on children with cancer not undergoing BMT, gastrostomy site infections made up 30.5\% ( $n=201)$ of complication episodes, occurring in $42.7 \%(n=96)$ of patients. In the two BMT studies, ${ }^{22,24}$ gastrostomy site infections made up $100 \%(n=14)$ of complication episodes, occurring in $28.4 \%(n=14)$ of patients. In all cases, infections were successfully treated using oral, local, or intravenous antibiotics.
Peritonitis occurred in $1.2 \%^{36}$ and $4.0 \%^{43}$ of total episodes, and abdominal wall abscess occurred in $4.0 \% 36,43$ of total episodes. Gastrostomy removal was required because of infection in $2.3 \%-27.3 \%{ }^{24,27}$ of patients, the latter being one of the BMT studies. No child required gastrostomy removal because of infection in the other BMT study. ${ }^{22}$ Infections were more common after 1 month after gastrostomy insertion (31 episodes vs 11 within the first month) $)^{25}$ and occurred, on average, $1.5^{26}$ and $5.2^{45}$ months postinsertion. All infections occurred within 2 months of placement in another study. ${ }^{24}$

Three studies compared complications between children with gastrostomy for cancer/BMT and those with neurological disability ${ }^{17,24,39}$ Whereas significantly higher inflammatory episodes were found in one study $(38.7 \%$ total episodes oncology vs $17.5 \%$ neurology group, $P=$ $.049)^{17}$ and infections in another $(36.5 \%$ in BMT vs $3.3 \%$ comparator group, $P=.01),{ }^{24}$ no difference in infection rates were seen in other studies. ${ }^{17,39}$ No differences between groups were seen for other complications, including leakage, granuloma, ${ }^{17,39}$ and material complications. ${ }^{17}$

\section{Insertion technique and device}

Three studies ${ }^{41,42,44}$ found no difference between gastrostomy insertion technique and type or number of complications, yet one ${ }^{25}$ found significantly more infections with open vs endoscopic/laparoscopic techniques $(P=$ 
.003). Local infections were more frequent with a button than with balloon devices $(P=.05),{ }^{42}$ whereas another study reported no significant difference between total complication episodes with button devices (64.2\%) vs tubes $(52.3 \%) .^{25}$

\section{Nutrition outcomes}

Height $z$-score decreased in two studies, from +0.8 at diagnosis to -0.2 at end of follow-up ( $P$-value not reported $)^{17}$ and from +0.03 at gastrostomy placement to -0.31 year after ( $P$-value not reported). ${ }^{38} \mathrm{~A}$ significant increase in mean weight from gastrostomy placement to 1 month $(P=.018)$ and 3 months postplacement $(P<.0001)$ was shown in one study, ${ }^{27}$ with others showing weight gains in $42.4 \%-73.0 \%^{17,42}$ of patients during gastrostomy feeding ${ }^{42}$ and $73 \%$ of patients 3 months after placement. ${ }^{40}$ Gastrostomy feeding also facilitated weight gain in malnourished children, with $50 \%$ and $77 \%$ gaining weight at 1 and 3 months postplacement, respectively, ${ }^{27}$ and $60 \%$ reaching ideal body weight after 5 months. ${ }^{41}$ Weight $z$-score significantly increased from gastrostomy placement to $1(P=.04),{ }^{38} 2(P=.039),{ }^{38}$ and $6(P<.05)^{45}$ months postplacement; however, a nonsignificant change was seen at 6 months postplacement $(P=.054)$ in another study. ${ }^{26}$

Two studies ${ }^{22,36}$ showed stability of nutrition status via gastrostomy and NGT but no difference between either on change in weight $z$-scores from BMT admission to discharge $(P=.379)^{22}$ and body mass index (BMI) $z$-scores from diagnosis to 6-12 months after treatment completion $(P=.09) .{ }^{36}$ Children fed via gastrostomy, compared with those fed via NGT, did not require parenteral nutrition as often during BMT admission $(P=.049) .{ }^{22}$ However, for children that needed parenteral nutrition, one study found similar duration between children fed via gastrostomy and those fed via NGT $(P=.140),{ }^{22}$ whereas another showed shorter duration during cancer treatment for those fed via gastrostomy rather than via NGT $(P=.0038) .{ }^{36}$

One BMT study found no difference between children fed via gastrostomy and those fed via NGT on the change in serum albumin level throughout admission or in the number of patients developing hypoalbuminemia $\leq 30 \mathrm{~g} / \mathrm{L}$ ( $88.9 \%$ vs $84.6 \%$, respectively; $P=.620) .{ }^{22}$ Nutrition outcomes are summarized in Table 3.

\section{Clinical outcomes}

Two studies found no difference in the length of admission for children fed via gastrostomy vs those fed via NGT-one during BMT ( 45 vs 46 days respectively, $P=.625)^{22}$ and the other on an average length of admission throughout can- cer treatment pregastrostomy and postgastrostomy (9.1 vs 8.2 days, respectively; no $P$-value reported). ${ }^{43}$ No significant differences were found between gastrostomy vs NGT on day 100 in overall mortality ( $0 \%$ vs $5.5 \%$, respectively; $P=.081)^{22}$, nonrelapse mortality (0\% vs $4.4 \%$, respectively; $P=.120),{ }^{22}$ or event-free survival at 4 years (74\% vs $85 \%$, respectively; $P=.31) .{ }^{36}$ One BMT study found no difference between children fed via gastrostomy and those fed via NGT on GvHD grades III-IV (3.7\% vs 6.6\%, respectively; $P=.664)$ and gut GvHD (3.7\% vs 9.9\%, respectively; $P=$ .191). ${ }^{22}$ Clinical outcomes are summarized in Table 3.

\section{DISCUSSION}

We present the first systematic review investigating the incidence and type of complications and outcomes of gastrostomy use in children with cancer, including those undergoing BMT. Gastrostomies were associated with frequent complications in $~ 50 \%$ of children ( $75 \%$ being minor, $25 \%$ major) and stabilization or improvement in nutrition status for the majority of children.

\section{Complications}

The classification of complications as minor or major was nonstandardized, leading to discrepancies between studies. Any infection was classified as minor in three studies $^{26,43,44}$ and major in two ${ }^{25,40}$; if treated with intravenous antibiotics, infection was classified as minor ${ }^{36}$ in one study yet major in others. ${ }^{17,22,24}$ In immunocompromised children, even a local infection could be viewed as a major event. ${ }^{24}$ Other studies defined major complications by unplanned events-for example, a hospital admission or interventional procedure. ${ }^{17,42,44}$ Different definitions have led to variations in major complications from $6.1 \%-17.5 \%$ in noncancer populations. ${ }^{46}$

The most frequent complications were inflammation and infection of the gastrostomy site, comprising 40\%$50 \%$ of total episodes. Significantly more infections were seen in 11 children undergoing BMT compared with those not undergoing BMT. ${ }^{24}$ Comparatively, no difference was seen in two studies with a combined sample of 101 undergoing cancer treatment (without BMT), compared with neurologically impaired children, ${ }^{17,39}$ and a further study found five children with BMT had no more complications than their wider population. ${ }^{26}$ As with the classification of complications, the differentiation between local infection and inflammation at the gastrostomy site also varied between studies. Some used a positive culture for microorganisms to confirm an infection, ${ }^{26,27,38,41,42,45}$ with others using clinician diagnosis alone to make the 
TA B LE 3 Nutrition and clinical outcomes of gastrostomy feeding

\begin{tabular}{|c|c|c|c|}
\hline Outcomes and authors & Time period & Results & $\boldsymbol{P}$ \\
\hline \multicolumn{4}{|l|}{ Weight-for-height $z$-score } \\
\hline $\operatorname{Schmitt}(2012)^{17}$ & Placement to end of enteral nutrition & +0.8 & Not reported \\
\hline \multicolumn{4}{|l|}{ Weight-for-age $z$-score } \\
\hline Evans $(2019)^{22}$ & BMT admission to discharge & Gastrostomy: 0.0 ; nasogastric: -0.1 & .379 \\
\hline \multirow[t]{2}{*}{ Parbhoo $(2011)^{45}$} & Placement to 5 months after & Maintained (no data) & Not reported \\
\hline & Removal to 6 months after & Increased -1.04 to -0.53 & $<.05$ \\
\hline \multirow[t]{3}{*}{ Skolin $(2002)^{38}$} & Placement to 1 month after & +0.03 & .04 \\
\hline & Placement to 2 months after & +0.11 & .039 \\
\hline & Placement to 3, 6, 12 months after & $-0.04,-0.12,+0.24$ & Not reported \\
\hline Pedersen $(1999)^{26}$ & Placement to 6 months after & +0.3 & .054 \\
\hline \multicolumn{4}{|l|}{ Weight, kg } \\
\hline $\operatorname{Schmitt}(2012)^{17}$ & Placement to end of enteral nutrition & $73.0 \%$ gained, $4.1 \%$ maintained, $23.0 \%$ lost & Not reported \\
\hline Richioud (2015) ${ }^{40}$ & Gastrostomy placement to 1 month after & $64.0 \%$ gained, $18.0 \%$ maintained, $9.0 \%$ lost & Not reported \\
\hline \multirow[t]{2}{*}{ Barron $(2000)^{27}$} & Gastrostomy placement to 1 month after & $38.6 \%$ gained, $54.0 \%$ maintained, $6.8 \%$ lost & .018 \\
\hline & Placement to 3 months after & $69.2 \%$ gained, $28.2 \%$ maintained, $2.6 \%$ lost & $<.0001$ \\
\hline Mathew $(1996)^{42}$ & During gastrostomy feeding & $\begin{array}{l}\text { 42.4\% gained, } 39.0 \% \text { maintained, } 18.0 \% \\
\text { lost }\end{array}$ & Not reported \\
\hline Aquino $(1995)^{41}$ & Placement to removal & Increase $12.9 \%$ desirable body weight & Not reported \\
\hline \multicolumn{4}{|l|}{ BMI $z$-score } \\
\hline \multirow[t]{2}{*}{ Henry $(2017)^{36}$} & Diagnosis to end of treatment & Gastrostomy: $-0.09 ;$ nasogastric: -0.13 & $>.05$ \\
\hline & Diagnosis to 6-12 months after treatment & Not reported & .09 \\
\hline \multicolumn{4}{|l|}{ Height $z$-score } \\
\hline Schmitt $(2012)^{17}$ & Diagnosis to end of follow-up & Decreased +0.8 to -0.2 & Not reported \\
\hline Skolin $(2002)^{38}$ & Gastrostomy placement to 1 year after & Decreased +0.03 to -0.3 & Not reported \\
\hline \multicolumn{4}{|l|}{ Survival } \\
\hline \multirow[t]{2}{*}{ Evans $(2019)^{22}$} & Overall survival 100 days post-BMT & Gastrostomy: 0\%; nasogastric: $5.5 \%$ & .081 \\
\hline & Nonrelapse mortality 100 days post-BMT & Gastrostomy: $0 \%$; nasogastric: $4.4 \%$ & .120 \\
\hline Henry $(2017)^{36}$ & Event-free survival at 4 years & Gastrostomy: $74.0 \%$; nasogastric: $85.0 \%$ & .31 \\
\hline \multicolumn{4}{|l|}{ Length of admission } \\
\hline Evans $(2019)^{22}$ & Days between BMT to discharge & Gastrostomy: 45 days; nasogastric: 46 days & .625 \\
\hline Bakish $(2003)^{43}$ & Days per month & $\begin{array}{l}\text { Pregastrostomy: } 9.1 \text { days; } \\
\text { postgastrostomy: } 8.2 \text { days }\end{array}$ & $\begin{array}{l}\text { Not } \\
\text { significant }\end{array}$ \\
\hline \multicolumn{4}{|l|}{ Parenteral nutrition } \\
\hline \multirow[t]{2}{*}{ Evans $(2019)^{22}$} & Requirement during BMT admission & Gastrostomy: $68.5 \%$; nasogastric: $81.3 \%$ & .049 \\
\hline & Days provided during BMT admission & Gastrostomy: 31 days; nasogastric: 31 days & .140 \\
\hline Henry $(2017)^{36}$ & Days provided during admission & $\begin{array}{l}\text { Gastrostomy: } 5.8 \text { days; nasogastric: } 9.9 \\
\text { days }\end{array}$ & .0038 \\
\hline
\end{tabular}

Abbreviations: BMI, body mass index; BMT, bone marrow transplant.

distinction. ${ }^{17,22,24,25,36,39,43}$ Certainly, from included studies, the potential for episodes of infection and inflammation to be misclassified was a possibility. Distinguishing between gastrostomy site infection and inflammation can be challenging in clinical practice, particularly in some cases in which these complications may coexist.

Risks associated with infections included neutropenia. No association was found between absolute neutrophil count (ANC) at gastrostomy placement and infection in two studies, ${ }^{25,39}$ whereas another found $50 \%$ of patients were neutropenic (ANC < 1000) at placement. $^{24}$ At time of infection, neutropenia (ANC < 500-1500) was found in $71.4 \%-75 \%$ of patients ${ }^{24,38}$ and $23.8 \%-100 \%$ of episodes. ${ }^{25,26,41,45}$ Compared with those who are adequately nourished, malnutrition has also been associated with more frequent infection in pediatric cancer patients. ${ }^{4,45,47}$ However, one study found more gastrostomy infections in the BMT, compared 
with the comparator group (with gastrostomies but not undergoing BMT) but no difference between groups on weight $z$-scores, suggesting neutropenia was the main contributor. ${ }^{24}$

Because of mixed evidence, it is difficult to draw conclusions regarding the timing of complications, gastrostomy devices, and insertion techniques. About 20\%$50 \%$ of complications were seen within the first month after gastrostomy placement ${ }^{24,25,39,45}$ and $30 \%-80 \%$ after 1 month. . $5,39,45$ Local infections were more frequent with buttons than with tube devices in one study $^{42}$; however, another reported no difference. ${ }^{25}$ Open rather than endoscopic/laparoscopic technique was associated with infections in one study, ${ }^{25}$ although three found no difference. ${ }^{41,42,44}$ This supports a recent systematic review that found no difference between these insertion techniques in children. ${ }^{48}$

\section{Outcomes}

Gastrostomy feeding was associated with improvement or stabilization of nutrition status in $77 \%-92.7 \%$ of children, with 50\%-77\% of malnourished children gaining or achieving ideal body weight ${ }^{27,41}$; these are important results given that malnourished children have associations with lower survival rates. ${ }^{7}$ Equivalent changes in nutrition status were seen, compared with NGTs. ${ }^{22,36}$ However, in many studies, parenteral nutrition was provided alongside gastrostomy and NG feeding, ${ }^{22,26,27,36,38}$ which will have contributed to results. In the studies comparing gastrostomy vs NGT, gastrostomy use was associated with less parenteral nutrition during BMT admission ${ }^{22}$ and throughout cancer treatment, ${ }^{36}$ possibly owing to gastrostomies avoiding certain complications of NGT such as refusal and dislodgment with vomiting, ${ }^{18,22}$ suggesting that gastrostomy, rather than NG feeding, played a greater role in supporting nutrition status. However, it is not the gastrostomy, per se, that facilitates benefits to nutrition status, but enteral nutrition. Few studies detailed the volume and type of enteral nutrition provided. Proactive enteral nutrition, given systematically in one center compared with another where this was not initiated, has prevented rapid weight loss at the beginning of chemotherapy and stabilized weight during followup in children with bone tumors. ${ }^{17}$ Enteral nutrition initiated systematically on day 1 post-BMT, compared with parenteral nutrition initiated at the same time, has also reduced hospital stay and improved survival in BMT. ${ }^{49,50}$ Less parenteral nutrition with gastrostomy use has economic and safety implications, given the cost of parenteral nutrition $^{51}$ and avoidance of parenteral nutrition-related complications. ${ }^{19,52}$
Most studies assessed nutrition status using weight $^{17,22,27,38,40-42,45}$ and BMI, ${ }^{36}$ which have limitations in this population, as they can be distorted by tumor mass and hydration status. ${ }^{53}$ More sensitive methods, such as mid-upper arm circumference, which is independent of such confounding factors, ${ }^{7,53}$ should be used in future studies.

\section{Limitations}

Limitations of the evidence include retrospective designs, small samples, absence of randomization, lack of comparator groups (meaning meta-analyses were not conducted), and heterogeneity regarding populations, gastrostomy devices and insertion techniques, classification of complications, and varying follow-up. Within studies, there was also an absence of subgroup analysis of complications and outcomes specific to underlying cancer diagnosis and treatment modality other than BMT. Consequently, the level of evidence varied, with five included studies judged at serious risk of bias and poor quality. However, the majority were judged as better quality, with the remaining 11 studies considered at moderate risk of bias to good quality. Nevertheless, this review provides the most accurate estimation of gastrostomy complications and outcomes in this population based on the currently best available evidence.

\section{Implications for practice}

In agreement with other authors, ${ }^{17,27,36,38,41-43}$ this review suggests gastrostomy use is relatively safe and associated with mostly minor complications and improvements in nutrition status. Consequently, we recommend gastrostomies could be placed, following careful consideration, prophylactically at diagnosis in specifically selected children-for example, those with existing malnutrition and diagnoses of high nutrition risk, as well as in preparation for intensive treatment protocols such as BMT, ${ }^{54}$ for which severe mucositis and long-term enteral nutrition are anticipated, and improvements might be seen in quality of life. Even though gastrostomies are more invasive than NGTs, the aesthetic benefit has been shown to aid acceptability to overcome resistance to tube feeding ${ }^{42,43}$ and relieve stress, enabling families to concentrate on others aspects of life. ${ }^{26,38}$ Proactive placement enables the wound to heal before neutropenia, and enteral nutrition to begin at the earliest need. Given many complications that might have been avoided with better care, ${ }^{26,38}$ families should receive gastrostomy education, and children should 
be monitored closely during neutropenia to minimize complications.

\section{Implications for research}

Further research should include prospective, multicenter trials to obtain adequately powered samples and provide higher-quality evidence. Gastrostomy complications should be compared between children with cancer and noncancer populations, with neurologically impaired children considered the "reference population."17,55 Randomizing such studies would not be possible, but uniform methods for classifying complications and consistent time points for follow-up should be applied. Future research should also investigate gastrostomy education strategies. Although education protocols have shown improvements in care in a preprotocol vs postprotocol evaluation, they have not shown reductions in complications, ${ }^{56}$ which future research should prioritize. Gastrostomy outcomes should be compared within cancer/BMT populations but including children fed via other routes, such as NGTs and parenteral nutrition. These trials should be randomized, detail the nutrition support strategy, and investigate outcomes, including nutrition intake and status (using sensitive measures), quality of life, and economic evaluation.

\section{CONCLUSION}

In conclusion, this review has shown gastrostomy use in children with cancer, including those undergoing BMT, is relatively safe, associated with frequent but mostly minor complications, and effective in stabilizing or improving nutrition status throughout treatment. The decision to place a gastrostomy requires careful consideration of the risks and benefits, including the complications and outcomes reported here, as well as the nutrition risk of the child's diagnosis, treatment intensity, nutrition status at diagnosis, expected length of enteral nutrition, and quality of life.

\section{ACKNOWLEDGMENTS}

This review presents independent research funded by the National Institute for Health Research (NIHR) (Clinical Doctoral Research Fellowship, project reference NIHR300554). This research was also supported in part by the NIHR Great Ormond Street Hospital Biomedical Research Centre. The views expressed are those of the author(s) and not necessarily those of the NHS, the NIHR, or the Department of Health and Social Care.

\section{CONFLICT OF INTEREST}

None declared.

\section{FUNDING INFORMATION}

This review presents independent research funded by the National Institute for Health Research (NIHR) (Clinical Doctoral Research Fellowship, project reference NIHR300554). This research was also supported in part by the NIHR Great Ormond Street Hospital Biomedical Research Centre.

\section{AUTHOR CONTRIBUTIONS}

James Evans contributed to the conception and design of the research; Breeana Gardiner, Dan Green, Faith Gibson, Graeme O'Connor, and Julie Lanigan contributed to the design of the research; James Evans, Breeana Gardiner, Dan Green, Faith Gibson, Graeme O'Connor, and Julie Lanigan contributed to the acquisition and analysis of the data; James Evans, Breeana Gardiner, Dan Green, Faith Gibson, Graeme O'Connor, and Julie Lanigan contributed to the interpretation of the data; and James Evans drafted the manuscript. All authors critically revised the manuscript, agree to be fully accountable for ensuring the integrity and accuracy of the work, and read and approved the final manuscript.

\section{O R C I D}

James Evans MRes (D) https://orcid.org/0000-0003-23838984

\section{REFERENCES}

1. Brinksma A, Huizinga G, Sulkers E, Kamps W, Roodbol P. Malnutrition in childhood cancer patients: a review on its prevalence and possible causes. Crit Rev Oncol Hematol. 2012;83(2):249-275.

2. Sala A, Pencharz P, Barr RD. Children, cancer, and nutrition - a dynamic triangle in review. Cancer. 2004;100(4):677-687.

3. Brinksma A, Sanderman R, Roodbol PF, et al. Malnutrition is associated with worse health-related quality of life in children with cancer. Support Care Cancer. 2015;23(10):3043-3052.

4. Loeffen EAH, Brinksma A, Miedema KGE, de Bock GH, Tissing WJE. Clinical implications of malnutrition in childhood cancer patients-infections and mortality. Support Care Cancer. 2015;23(1):143-150.

5. Thompson PA, Rosner GL, Matthay KK, et al. Impact of body composition on pharmacokinetics of doxorubicin in children: a glaser pediatric research network study. Cancer Chemother Pharmacol. 2009;64(2):243-251.

6. Taj MM, Pearson ADJ, Mumford DB, Price L. Effect of nutritional status on the incidence of infection in childhood cancer. Pediatr Hematol Oncol. 1993;10(3):283-287. 
7. Sala A, Rossi E, Antillon F, et al. Nutritional status at diagnosis is related to clinical outcomes in children and adolescents with cancer: a perspective from Central America. Eur J Cancer. 2012;48(2):243-252.

8. Kerby EH, Li Y, Getz KD, et al. Nutritional risk factors predict severe acute graft-versus-host disease and early mortality in pediatric allogeneic hematopoietic stem cell transplantation. Pediatr Blood Cancer. 2018;65(2).

9. Ward E, Evans J. Childhood cancers and immunodeficiency syndromes. In: Shaw V, ed. Clinical Paediatric Dietetics. 5th ed. Wiley; 2020:371-390.

10. Walrath M, Bacon C, Foley S, Fung HC. Gastrointestinal side effects and adequacy of enteral intake in hematopoietic stem cell transplant patients. Nutr Clin Pract. 2015;30(2):305-310.

11. Kestler SA, Lobiondo-Wood G. Review of symptom experiences in children and adolescents with cancer. Cancer Nurs. 2012;35(2):31-49.

12. Cameron N, Demerath EW. Critical periods in human growth and their relationship to diseases of aging. Yearb Phys Anthropol. 2002;45:159-184.

13. August D, Huhmann M; American Society of Parenteral and Enteral Nutrition (ASPEN) Board of Directors. A.S.P.E.N. clinical guidelines: nutrition support therapy during adult anticancer treatment and in hematopoietic cell transplantation. JPEN J Parenter Enter Nutr. 2009;33(5):472-500.

14. Muscaritoli, M., Arends, J., Bachmann, P., Baracos, V., et al. 2017. ESPEN practical guideline: Clinical Nutrition in cancer. Clinical Nutrition, 40(5), 2898-2913.

15. Trimpe K, Shaw MR, Wilson M, Haberman MR. Review of the effectiveness of enteral feeding in pediatric oncology patients. $J$ Pediatr Oncol Nurs. 2017;34(6):439-445.

16. Den Broeder E, Lippens RJJ, Van't Hof MA, et al. Effects of nasogastric tube feeding on the nutritional status of children with cancer. Eur J Clin Nutr. 1998;52(7):494-500.

17. Schmitt F, Caldari D, Corradini N, et al. Tolerance and efficacy of preventive gastrostomy feeding in pediatric oncology. Pediatr Blood Cancer. 2012;59(5):874-880.

18. Trehan A, Viani K, da Cruz LB, Sagastizado SZ, Ladas EJ. The importance of enteral nutrition to prevent or treat undernutrition in children undergoing treatment for cancer. Pediatr Blood Cancer. 2020;67(S3):1-8.

19. Evans JC, Hirani SP, Needle JJ. Nutritional and posttransplantation outcomes of enteral versus parenteral nutrition in pediatric hematopoietic stem cell transplantation: a systematic review of randomized and non-randomized studies. Biol Blood Marrow Transplant. 2019;25(8):e252-e259

20. Thompson JL, Duffy J. Nutrition support challenges in hematopoietic stem cell transplant patients. Nutr Clin Pract. 2008;23(5):533-546.

21. Mcgrath KH, Hardikar W. Gastrostomy tube use in children with cancer. Pediatr Blood Cancer. 2019;66(7):1-7.

22. Evans J, Needle JJ, Hirani SP. Early outcomes of gastrostomy feeding in paediatric allogenic bone marrow transplantation: a retrospective cohort study. Clin Nutr ESPEN. 2019;31:71-79.

23. Evans J. How patients can enhance research into feeding children during bone marrow transplantation. Nurs Child Young People. 2019;31(4):19-19.

24. Kaur S, Ceballos C, Bao R, Pittman N, Benkov K. Percutaneous endoscopic gastrostomy tubes in pediatric bone mar- row transplant patients. J Pediatr Gastroenterol Nutr. 2013;56(3): 300-303.

25. Fernandez-Pineda I, Sandoval J, Jones R, et al. Gastrostomy complications in pediatric cancer patients: a retrospective single-institution review. Pediatr Blood Cancer. 2016;63(7):12501253.

26. Pedersen AMB, Kok K, Petersen G, Nielsen OH, Michaelsen KF, Schmiegelow K. Percutaneous endoscopic gastrostomy in children with cancer. Acta Paediatr. 1999;88(8):849-852.

27. Barron MA, Duncan DS, Green GJ, et al. Efficacy and safety of radiologically placed gastrostomy tubes in paediatric haematology/oncology patients. Med Pediatr Oncol. 2000;34(3):177-182.

28. Shamseer L, Moher D, Clarke M, et al. Preferred reporting items for systematic review and meta-analysis protocols (PRISMA-P) 2015: elaboration and explanation. BMJ. 2015;349:1-25.

29. Liberati A, Altman DG, Tetzlaff J, et al. The PRISMA statement for reporting systematic reviews and meta-analyses of studies that evaluate health care interventions: explanation and elaboration. PLoS Med. 2009;6(7):e1000100.

30. Townley A, Wincentak J, Krog K, Schippke J, Kingsnorth S. Paediatric gastrostomy stoma complications and treatments: a rapid scoping review. J Clin Nurs. 2017;27(7-8):1369-1380.

31. Sandberg F, Viktorsdóttir MB, Salö M, Stenström P, Arnbjörnsson E. Comparison of major complications in children after laparoscopy-assisted gastrostomy and percutaneous endoscopic gastrostomy placement: a meta-analysis. Pediatr Surg Int. 2018;34(12):1321-1327.

32. Covidence. Accessed September 18, 2020. www.covidence.org

33. Mendeley Desktop. Version 1.19.4. Mendeley Ltd; 2019. Accessed September 18, 2020. https://www.mendeley.com/referencemanagement/mendeley-desktop.

34. Sterne JA, Hernán MA, Reeves BC, et al. ROBINS-I: a tool for assessing risk of bias in non-randomised studies of interventions. BMJ. 2016;355:i4919.

35. National Institutes of Health (NIH). Quality assessment tool for before-after (pre-post) studies with no control group. Accessed September 28, 2020. https://www.nhlbi.nih.gov/health-topics/ study-quality-assessment-tools

36. Henry C, Dumoucel S, Taque S, et al. Assessment of early gastrostomy in the treatment of primary malignant bone tumors in children. A report from the French children's oncology study group GOCE. Rev d'Oncologie Hematol Pediatr. 2017;5(1): 10-20.

37. Arnbjörnsson E, Larsson LT, Lindhagen T. Complications of laparoscopy-aided gastrostomies in pediatric practice. J Pediatr Surg. 1999;34(12):1843-1846.

38. Skolin I, Hernell O, Larsson MV, Wahlgren C, Wahlin YB. Percutaneous endoscopic gastrostomy in children with malignant disease. J Pediatr Oncol Nurs. 2002;19(5):154-163.

39. Arnbjornsson E, Backman T, Morse H, Berglund Y, Kullendorff CM, Lovkvist H. Complications of video-assisted gastrostomy in children with malignancies or neurological diseases. Acta Paediatr. 2006;95(4):467-470.

40. Richioud B, Louazon T, Beji H, et al. De novo radiologic placement of button gastrostomy: a feasibility study in children with cancer. Pediatr Radiol. 2015;45(13):1957-1963.

41. Aquino V, Sirynl C, Hagg R, McHard K, Prestridge L, Sandler E. Enteral nutrition support by gastrostomy tube in children with cancer. J Pediatr. 1995;127(1):58-62. 
42. Mathew $\mathrm{P}$, Bowman L, Williams $\mathrm{R}$, et al. Complications and effectiveness of gastrostomy feedings in pediatric cancer patients. J Pediatr Hematol Oncol. 1996;18(1):81-85.

43. Bakish J, Hargrave D, Tariq N, Laperriere N, Rutka JT, Bouffet E. Evaluation of dietetic intervention in children with medulloblastoma or supratentorial primitive neuroectodermal tumors. Cancer. 2003;98(5):1014-1020.

44. Hamilton EC, Curtin T, Slack RS, et al. Surgical feeding tubes in pediatric and adolescent cancer patients: a single-institution retrospective review. J Pediatr Hematol Oncol. 2017;39(7):e342e348.

45. Parbhoo D, Tiedemann K, Catto-Smith A. Clinical outcome after percutaneous endoscopic gastrostomy in children with malignancies. Pediatr Blood Cancer. 2011;56(7):1146-1148.

46. Hansen E, Qvist N, Rasmussen L, Ellebæk MB. Postoperative complications following percutaneous endoscopic gastrostomy are common in children. Acta Paediatr. 2017;106(7): 1165-1169.

47. Roberts SR, Miller JE. Success using PEG tubes in marrow transplant recipients. Nutr Clin Pract. 1998;13(2):74-78.

48. Baker L, Beres AL, Baird R. A systematic review and metaanalysis of gastrostomy insertion techniques in children. J Pediatr Surg. 2015;50(5):718-725.

49. Azarnoush S, Bruno B, Beghin L, et al. Enteral nutrition: a first option for nutritional support of children following allo-SCT. Bone Marrow Transplant. 2012;47(9):1191-1195.

50. Gonzales F, Bruno B, Alarcón Fuentes M, et al. Better early outcome with enteral rather than parenteral nutrition in children undergoing MAC allo-SCT. Clin Nutr. 2017;37(6): 2113-2121.

51. Cangelosi MJ, Auerbach HR, Cohen JT. A clinical and economic evaluation of enteral nutrition. Curr Med Res Opin. 2011;27(2):413-422.
52. McGrath KH, Evans V, Yap J. Indications and patterns of use for parenteral nutrition in pediatric oncology. JPEN J Parenter Enter Nutr. 2019;44(4):632-638.

53. Viani K, Trehan A, Manzoli B, Schoeman J. Assessment of nutritional status in children with cancer: a narrative review. Pediatr Blood Cancer. 2020;67 Suppl 3:e28211.

54. Selwood K, Ward E, Gibson F. Assessment and management of nutritional challenges in children's cancer care: a survey of current practice in the United Kingdom. Eur J Oncol Nurs. 2010;14(5):439-446.

55. El-Matary W. Percutaneous endoscopic gastrostomy in children. Can J Gastroenterol Hepatol. 2008;22(12):993-998.

56. Schweitzer M, Aucoin J, Docherty SL, Rice HE, Thompson J, Sullivan DT. Evaluation of a discharge education protocol for pediatric patients with gastrostomy tubes. J Pediatr Heal Care. 2014;28(5):420-428.

\section{SUPPORTING INFORMATION}

Additional supporting information may be found online in the Supporting Information section at the end of the article.

How to cite this article: Evans J, Gardiner B, Green D, Gibson F, O'Connor G, Lanigan J. Systematic review of gastrostomy complications and outcomes in pediatric cancer and bone marrow transplant. Nutr. Clin. Pract. 2021;1-13.

https://doi.org/10.1002/ncp.10724 